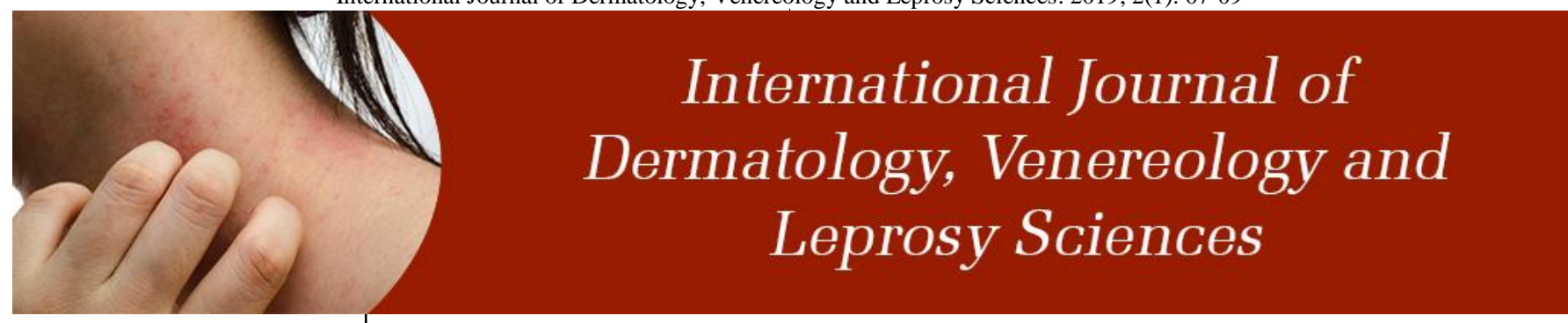

E-ISSN: 2664-942X

P-ISSN: 2664-9411

Derma 2019; 2(1): 07-09

Received: 06-11-2018

Accepted: 09-12-2018

\section{Dr. Rajiv Kumar}

Department of Dermatology,

King Edward Memorial

Hospital and Seth Gordhandas

Sunderdas Medical College,

Mumbai, Maharashtra, India

\section{Correspondence}

Dr. Rajiv Kumar

Department of Dermatology,

King Edward Memorial

Hospital and Seth Gordhandas

Sunderdas Medical College,

Mumbai, Maharashtra, India

\title{
Evaluation of acne vulgaris in females of all age groups- A clinical study
}

\section{Dr. Rajiv Kumar}

DOI: https://doi.org/10.33545/26649411.2019.v2.i1a.17

Abstract

Background: Acne vulgaris is a common skin disease affecting adolescents and young adults worldwide.

Materials \& Methods: The present study was conducted on 68 females patients. A thorough clinical examination was done in all patients and site of lesion and grade of acne were recorded.

Results: Age group 10-20 years had 25, 20-30 years had 22, 30-40 years had 15 and >40 years had 6 patients. The difference was significant $(P<0.05)$. Common site was face $\mathrm{n} 32$, back in 10 , face and chest in $12 \&$ chest and back in 14 cases. The difference was significant $(P<0.05)$. Grade I was seen in 10 , II in 28 , III in 26 and IV in 4 . The difference was significant $(P<0.05)$.

Conclusion: Maximum cases of Acne were seen in 10-20 years and face was the commonest site of occurrence.

Keywords: Acne vulgaris, Chest, Face

\section{Introduction}

Acne vulgaris is a common skin disease affecting adolescents and young adults worldwide. It affects over $90 \%$ of males and $80 \%$ of females in all ethnic groups. Acne affects $9.4 \%$ of the world's population, making it the most prevalent worldwide disease. Acne significantly affects physical and psychosocial well-being. The pathogenesis of acne vulgaris is multifactorial, and hormones, sebum production and bacterial colonization playing major roles ${ }^{[1]}$.

Acne lesions are typically classified as non inflammatory (open and closed comedones) or inflammatory (papules and pustules). Seborrhoea, or grease production, is also a feature. Scarring is often present following inflammation. The pathophysiological events occurring in acne are also relatively well studied. Lesions start when keratinocytes lining the hair follicle desquamate creating a microcomedone ${ }^{[2]}$.

Psychological stress has also been identified amongst the causative factors that exacerbate acne. It has been thought that psychological stress can alter the immune functions of the skin 6 and cutaneous barrier function ${ }^{[3]}$. But there are not many studies done to establish the relationship.

There are various factors that contribute to the appearance and severity of acne. Furthermore, acne has an impact on personal body image and can affect the quality of life (QOL). The impact of acne on QOL and the factors which influence QOL of patients with acne is not well characterized. Stresses can be external (from environment, psychological, or social situations) 3 or internal (illness or from a medical procedure). Stress affects $15-70 \%$ of population depending upon various sociodemographic parameters ${ }^{[4]}$. The present study was conducted to determine cases of Acne vulgaris in females.

\section{Materials \& Methods}

The present study was conducted in the department of Dermatology. It comprised of 68 females patients. All participants were informed regarding the study and written consent was obtained. The study was approved from institutional ethical committee.

Patient data such as name, age, gender etc. was recorded. A thorough clinical examination was done in all patients and site of lesion and grade of acne were recorded. Results thus obtained were subjected to statistical analysis. $\mathrm{P}$ value less than 0.05 was considered 
significant.

\section{Results}

Table I: Age wise distribution of patients

\begin{tabular}{|c|c|c|}
\hline Age groups (Years) & Number & P value \\
\hline $10-20$ & 25 & \multirow{2}{*}{0.02} \\
\hline $20-30$ & 22 & \\
\hline $30-40$ & 15 & \\
\hline$>40$ & 6 & \\
\hline
\end{tabular}

Table I shows that age group 10-20 years had 25, 20-30 years had 22, 30-40 years had 15 and $>40$ years had 6 patients. The difference was significant $(P<0.05)$.

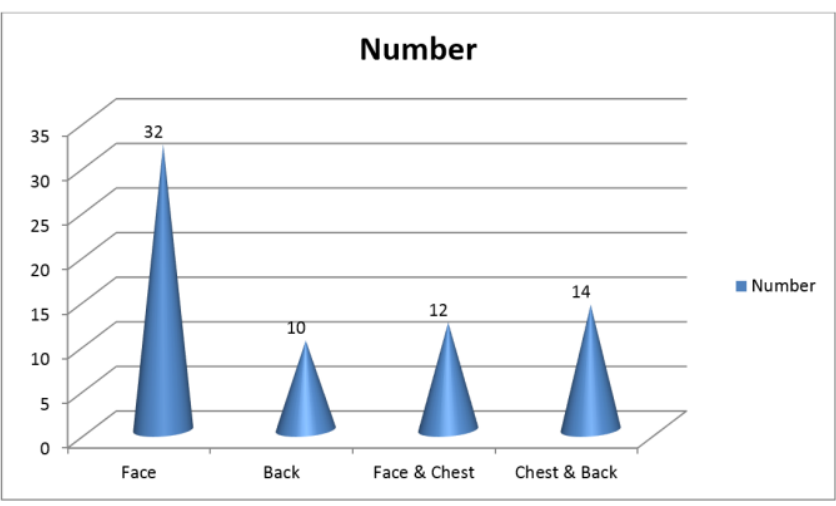

Graph 1: Site of Acne in patients

Graph I shows that common site was face n 32, back in 10 , face and chest in $12 \&$ chest and back in 14 cases. The difference was significant $(P<0.05)$.

Table II: Grading of Acne vulgaris

\begin{tabular}{|c|c|c|}
\hline Grading & Number & \multirow{2}{*}{ P value } \\
\hline I & 10 & \multirow{2}{*}{0.05} \\
\hline II & 28 & \\
\hline III & 26 & \\
\hline IV & 4 & \\
\hline
\end{tabular}

Table II shows that grade I was seen in 10, II in 28, III in 26 and IV in 4 . The difference was significant $(P<0.05)$.

\section{Discussion}

There are a number of proposed mechanisms of why stress aggravates acne. In adult women with acne, chronic stress increases the secretion of adrenal androgens and results in sebaceous hyperplasia. Activation of the hypothalamopituitary-adrenal (HPA) axis is the main adaptive response to systemic stress ${ }^{[5]}$. In response to emotional stress, the HPA axis activates increased levels of cortisol release. Corticotropin releasing hormone $(\mathrm{CRH})$ is the most proximal element of the HPA axis. CRH acts as a central coordinator for neuroendocrine and behavioral responses to stress. CRH stimulates sebaceous gland lipid production and steroidogenesis, which contributes to acne. Studies have also shown an increase of CRH expression in the sebaceous glands of acne-involved skin, compared to a low expression in normal skin ${ }^{[6]}$.

This up regulation of $\mathrm{CRH}$ expression in acne- involved skin may influence the inflammatory processes that lead to stress induced acne lesions. CRH also induces cytokines IL6 and IL-11 production in keratinocytes, contributing to inflammation, which is regarded as a key component in the pathogenesis of acne. Peripheral nerves release the neuropeptide substance $\mathrm{P}$ or vaso-intestinal peptide in response to stress. Substance $\mathrm{P}$ stimulates the proliferation and differentiation of sebaceous glands and up regulates lipid synthesis in sebaceous cells. Also, psychological stress could delay wound healing up to $40 \%$, which could affect the repair of acne lesions [7]. The present study was conducted to determine cases of Acne vulgaris in females. We found that age group 10-20 years had 25, 20-30 years had 22, 30-40 years had 15 and $>40$ years had 6 patients.

Saboo et al. ${ }^{[8]}$ conducted a study to determine the relationship between stress and acne severity.

All the subjects were assessed on Perceived Stress Scale to assess the level of stress they may have felt over past one month. All the diagnosed cases of acne vulgaris were assessed by Global Acne Grading Scale (GAGS) to assess the severity of acne. The results indicate that there is positive correlation between stress \& acne. The cases showed high stress levels as compared to the controls which were statistically significant $(p<0.01)$. The severity of acne vulgaris was also seen significantly more in subjects suffering from high stress levels $(p<0.05)$.

We found that common site was face n 32 , back in 10 , face and chest in $12 \&$ chest and back in 14 cases. The difference was significant $(P<0.05)$. Grade I was seen in 10 , II in 28 , III in 26 and IV in 4 . The difference was significant $(P<$ $0.05)$.

Hazarika et al. ${ }^{[9]}$ found that the prevalence of acne among medical students was $58.8 \%$. Combined type of acne was the most common clinical presentation. Predictors of acne were stress $(P<0.001)$, menstrual flaring $(P<0.001)$, and family history of the condition $(P<0.01)$. Females with severe acne had the lowest score (worse QOL) of all QOL domains compared to others $(P<0.05)$. Most of QOL domains were lower among students with psychological problems, anxiety, and depression.

Lello et al. ${ }^{[4]}$ found that most cases $(64 \%)$ were between 15 and 20 years. Females (57\%) outnumbered males. Facial lesions (61.4\%) and grade II acne were most common. Mean DLQI score was 7.22. DLQI scores were statistically influenced by the age of the patient, duration and grade of acne, acne scar, and postacne hyperpigmentation. This study showed significant impairment of QoL in acne patients. Assurance and counseling along with early treatment of acne vulgaris are important to reduce disease related psychosocial sequelae and increase the efficacy of treatment.

\section{Conclusion}

Authors found that maximum cases of Acne were seen in 10-20 years and face was the commonest site of occurrence.

\section{References}

1. Suh DH, Kim BY, Min SU et al. A multicenter epidemiological study of acne vulgaris in Korea. Int $\mathrm{J}$ Dermatol. 2011; 50(6):673-681.

2. Chiu A, Chon SY, Kimball AB. The response of skin disease to stress: changes in the severity of acne vulgaris as affected by examination stress. Arch Dermatol. 2003; 139(7):897-900.

3. Yosipovitch G, Tang M, Dawn AG et al. Study of psychological stress sebum production and acne vulgaris in adolescents. Acta Derm Venereol 2007; 
87(2):135-139.

4. Kligman AM. Post-adolescent acne in women. Cutis 1991; 48(1):75-77.

5. Ganceviciene $\mathrm{R}$, Graziene $\mathrm{V}$, Fimmel $\mathrm{S}$ et al. Involvement of the corticotropin-releasing hormone system in the pathogenesis of acne vulgaris. $\mathrm{Br} \mathrm{J}$ Dermatol. 2009; 160(2):345-352.

6. Zouboulis CC, Seltmann $\mathrm{H}$, Hiroi $\mathrm{N}$ et al. Corticotropin-releasing hormone: an autocrine hormone that promotes lipogenesis in human sebocytes. Proc Natl Acad Sci U S A 2002; 99(10):7148-7153.

7. Aktan S, Ozmen E, Sanli B. Anxiety, depression, and nature of acne vulgaris in adolescents. Int J Dermatol 2000; 39:354-7.

8. Saboo AV, Agarwal NA. Study of correlation between stress and acne vulgaris in young unmarried females. J. Evid. Based Med. Healthc. 2019; 6(6):345-347.

9. Hazarika N, Rajaprabha RK. Assessment of life quality index among patients with acne vulgaris in a suburban population. Indian J Dermatol. 2016; 61:163-8.

10. Lello J, Pearl A, Arroll B, Yallop J, Birchall NM. Prevalence of acne vulgaris in Auckland senior high school students. N Z Med J. 1995; 108:287-9. 